Printed in Great Britain

\title{
The Fine Structure of the Zoospores and Cysts of Allomyces macrogynus
}

\author{
By E. P. HILL \\ Department of Biology, Macalester College, St Paul, Minnesota 55ror, U.S.A. \\ (Accepted for publication 9 December 1968) \\ SUMMARY \\ Zoospores and cysts of Allomyces macrogynus have been studied by elec- \\ tron microscopy. A membrane-bound nuclear cap filled with ribosomes sur- \\ rounded a single nucleus. Mitochondria and lipid bodies were found at the \\ periphery of the nuclear cap. The flagellum was attached close to the nucleus \\ by two tubular structures running anteriorly along both sides of the nuclear \\ cap. During encystment the axoneme was retracted, the nuclear cap dissected \\ with endoplasmic reticulum and the mitochondria increased in number and \\ started to elongate. Concentric granules and/or mitochondria were found \\ embedded in the nuclear cap.
}

\section{INTRODUCTION}

Examination of the posteriorly uniflagellate spores of the aquatic Phycomycetes by electron microscopy, primarily of thin sections, has revealed a structural organization from which generalizations can be made. The zoospores of Monoblepharella sp. (Fuller \& Reichle, 1968), Blastocladiella emersonii (Reichle \& Fuller, 1967), Rhizidiomyces apophysatus (Fuller \& Reichle, 1965) and Nowakowskiella profusa (Chambers, Markus \& Willoughby, 1967) all possess a ribosomal region (nuclear cap) closely associated with the single nucleus. The ribosomal region is membrane-bound in all except Rhizidiomyces apophysatus. They all possess a flagellum that in cross-section shows the typical $9+2$ fibrillar configuration, a kinetosome and centrioles. However, the details of nuclear-cap organization and flagellar anchorage vary from organism to organism. All are surrounded by a cell membrane not enveloped by a cell wall. Mitochondria, endoplasmic reticulum, vacuoles, vesicles and lipid bodies are structures typically found in all of these spores.

This work was undertaken to determine the fine structure of Allomyces macrogynus zoospores and cysts as a preliminary to a study of sporogenesis and spore germination. Similarities and differences between these structures and closely related aquatic Phycomycetes are pointed out.

\section{METHODS}

The organism was grown in Petri dishes filled with Emerson (I958) YpSs agar for 5 days at room temperature in complete darkness. The plates were flooded with sterile tap water and allowed to stand for $45 \mathrm{~min}$., after which the zoospores were in heavy concentration. The spores began to emerge from sporangia about $15^{-20} \mathrm{~min}$. after being flooded. The time from flooding the plates to fixation was about $55 \mathrm{~min}$. Spores prepared in this manner represented a heterogeneous population in which motile spores and those that had encysted were always present, although there were fewer encysted spores than motile spores.

Vol. 56, No. I, was issued I9 May 1969 
The spores were collected by centrifugation and fixed in $\mathrm{I} \% \mathrm{OsO}_{4}$ in $0 . \mathrm{I} \mathrm{M}$-cacodylate buffer at $\mathrm{pH} 7 \cdot 0$ for $45 \mathrm{~min}$. After fixation they were washed with cacodylate buffer, pelleted in I \% water agar and dehydrated in a series of ethanol-water mixtures. The pellets were embedded in Epon 8I 2 and sectioned with a Porter-Blum microtome employing glass knives. The sections were stained for $10 \mathrm{~min}$. in $3.5 \%$ aqueous uranyl acetate followed by lead citrate (Reynolds, 1963) and viewed with an RCA-EMU $3 \mathrm{G}$ electron microscope.

\section{RESULTS}

A near median section of a typical zoospore is shown in Pl. I, fig. I. Prominent among the features are the membrane-bound ribosomal region, herein referred to as the nuclear cap, a well-defined nucleus and a large nucleolus. A single large mitochondrion is usually found at the posterior end adjacent to the flagellum, with smaller mitochondria lining the periphery of the nuclear cap. Associated with most mitochondria is a small strand of endoplasmic reticulum. A few ribosomes are usually found lying between the strands of endoplasmic reticulum, Pl. 3, fig. 7. When the zoospore loses its motility it becomes spherical, the flagellum is retracted, the nuclear cap becomes dissected with endoplasmic reticulum and there is a proliferation of mitochondria; thus the motile spore develops into a cyst. A comparison of Pl. I, fig. I and 2 shows these changes, which take place in a period of $30 \mathrm{~min}$. in an aqueous environment.

Mitochondria, as shown in Pl. I, fig. I and Pl. 3, fig. 5, are spherical in the motile spore with inclusions that are of the approximate size and staining character of ribosomes in the nuclear cap. The close association of the large mitochondrion with the nucleus is shown in Pl. 2, fig. 4 with the nucleus extending into the mitochondrion, although both are separated by their own membranes. During the encystment period the mitochondria become more numerous and prior to germination of the cyst they are found not only around the nuclear cap but also at the periphery of the spore (Pl. I, fig. 2; Pl. 3, fig. 8). When the germ tube has just emerged few spherical and mostly elongated mitochondria are found (Hill, unpublished observations).

The endoplasmic reticulum as shown in Pl. 2, fig. 4 and Pl. 3, fig. 5 and 6 is closely associated with both the mitochondria and nuclear cap in the motile spore. In several instances the endoplasmic reticulum surrounding the nuclear cap extends around a mitochondrion.

Although the nuclear cap is bound by a double membrane that is continuous with the nuclear membrane, as shown by the arrow in Pl. 2, fig. 4, Pl. 4, fig. 9, and Pl. 5, fig. I5, it was seldom found to be homogeneous. In most cases mitochondria and/or other granules, as seen in Pl. I, fig. I and Pl. 4, fig. I I, appear to be embedded in it. Some of the endoplasmic reticulum that dissects the nuclear cap during encystment (Pl. I, fig. 2; and Pl. 2, fig. 3) appears to originate in the cap itself. As the spore progresses toward encystment dissection of the cap progresses so that ribosomal areas are cut off into smaller units each surrounded by a double membrane (Pl. 3, fig. 8). Plate 4, fig. 9 shows a large area of ribosomes cut off anterior to the nuclear cap. When the spore (now cyst) germinates the nuclear cap is no longer intact or recognizable as such and the ribosomes are dispersed throughout the germ tube and spore body (Hill, unpublished observations).

The single whiplash flagellum, posteriorly located and closely associated with the 
nuclear membrane as found in the motile spore, is shown in Pl. I, fig. I, Pl. 4, fig. 9, IO and P1. 5, fig. I3. The outer membrane is continuous with the cell membrane, which could account for the numerous spores observed with flagellar bulbs (P1. 5, fig. I5). The axoneme, as shown in P1. 4, fig. 9 and Io, appears to fuse with the outermost unit of the nuclear membrane. The kinetosome is not bound at the cell membrane by a terminal plate. A cross-section of an extended flagellum showing the $9+2$ configuration is shown in Pl. 5, fig. I6. A centriole, indicated by the arrow in Pl. 4, fig. I0, is attached to the kinetosome. The retracted axoneme observed around the inside periphery of the encysted spore can be seen in longitudinal section in Pl. 5, fig. I4. A cross-section of a retracted axoneme is shown in Pl. I, fig. 2, Pl. 3, fig. 8 and Pl. 5, fig. I7.

A unique inclusion found in most sections was a granule of concentric rings either embedded in or lying close to the nuclear cap. It was usually enclosed by a double membrane and usually surrounded by an electron-transparent space, as shown in Pl. 4, fig. II and I2.

Lipid bodies were present in both motile and encysted spores (PI. I, fig. I and 2), being more abundant in the latter than the former. They were usually found scattered around the periphery of the nuclear cap. Small vacuoles were always observed in the electron-transparent area found between the nuclear cap and cell membrane (PI. I, fig. 1).

\section{DISCUSSION}

The zoospores of Allomyces macrogynus are strikingly similar in gross morphology to the zoospores of Blastocladiella emersonii (Reichle \& Fuller, 1967; Lessie \& Lovett, 1968) because of the position of the nucleus and nuclear cap at the posterior end and ribosomal composition of the nuclear cap. However, they differ from $B$. emersonii zoospores in that they have several mitochondria, compared to one in $B$. emersonii, no structures corresponding to the gamma bodies in B. emersonii and lipid bodies scattered around the periphery of the nuclear cap as compared to the lipid sac of $B$. emersonii.

The nuclear cap is clearly a distinguishing feature of the Blastocladiella zoospore. If fine structure analysis is to be used not only for comparative purposes but as an aid to the establishment of relationships among aquatic Phycomycetes, then the nuclear cap would appear to be a logical structure for this purpose. No criteria have yet been established to indicate what constitutes a 'characteristic nuclear cap' of any order of the aquatic Phycomycetes. This is due, in part, to insufficient evidence based on electron-micrograph analysis and to the lack of the number of species in which the motile phase has been examined.

No evidence was obtained in this study to indicate, as Fuller \& Reichle (I968) suggested, that the membrane surrounding the nuclear cap is derived from the nuclear membrane even though as shown in Pl. 2, fig. 4 and Pl. 5, fig. 15 they are connected. Ribosomes in Allomyces macrogynus zoospores are not confined to the nuclear cap as they are in the fully developed zoospore of Blastocladiella emersonii (Fuller, 1966; Fuller \& Reichle, 1968) since they are also found surrounded by a double membrane as pockets and lying individually, unattached between strands of endoplasmic reticulum (Pl. 3, fig. 5, 6, 7 and Pl. 4, fig. 9). Dissection and subsequent disintegration of the nuclear cap is apparently a fundamental process that occurs during the develop- 
ment of flagellated fungal spores since it is known to occur in zygote formation in $A$. macrogynus (Blondell \& Turian, 1960), encysting zoospores of B. emersonii (Reichle \& Fuller, 1967), post-encysted zoospores of Rhizidiomyces apophysatus (Fuller \& Reichle, I965) and $A$. macrogynus zoospores (Pl. I, fig. 2; Pl. 2, fig. 3).

The centriole, indicated by the arrow in Pl. 4, fig. 10, does not show the striations characteristic of Blastocladiella emersonii (Reichle \& Fuller, 1967) or the gametes of Allomyces arbusculus (Renaud \& Swift, 1964) and A. macrogynus (Blondell \& Turian, 1960). The kinetosome does not have a well-defined terminal plate nor are microtubules present. These observations are probably indicative of developmental events that take place prior to flagellar withdrawal and subsequent encystment. Although Fuller (1966) mentioned the presence of microtubules in Rhizidiomyces apophysatus, B. emersonii and Monoblepharella sp. they were not observed in A. macrogynus zoospores and they were neither apparent nor mentioned by Renaud \& Swift (I964) for $A$. arbusculus gametes nor by Blondell \& Turian (1960) during gametogenesis of $A$. macrogynus. However, as seen in Pl. I, fig. I and Pl. 4, fig. 9, the kinetosome is connected to two tubular structures running on either side of the nuclear cap. These structures may function as microtubules in giving internal stability to the cell during the motile phase.

Two explanations of the disappearance of the flagella at the end of the motile phase are that they are cast off or resorbed (Kole, 1965). A third possible explanation, presented by evidence shown in Pl. I, fig. 2, Pl. 3, fig. 8 and Pl. 5, fig. $\mathrm{I}_{4}$ and $\mathrm{I} 5$, is that the cell membrane surrounding the axoneme is probably resorbed, while the axoneme is retracted at the termination of the motile phase. That the flagellar sheath found in Allomyces macrogynus zoospores is not withdrawn is evident by comparing Pl. 5, fig. I 3 and 14 and fig. 16 and 17. Several spores were seen with bulbs similar to that shown in Pl. 5, fig. I5, while in no instance was the cell membrane surrounding the axoneme observed attached to the cell membrane, except at the junction of the cell body. Although it is evident that the axoneme is withdrawn, no mechanism nor structure as to how this is accomplished is known.

The close association between the large mitochondrion, the nucleus (Pl. 2, fig. 4) and the flagellum (Pl. 4, fig. 9 and 10 ) suggests an intimate relationship between control and energy supply and utilization during the motile phase. This association is even more pronounced in Blastocladiella emersonii zoospores (Reichle \& Fuller, 1967), where the single mitochondrion is perforated through which passes the kinetosome. During the encystment period the mitochondria become more numerous (compare Pl. I, fig. I and 2), and are dispersed throughout the spore. In early germlings most of the mitochondria are elongate rather than spherical. These changes are apparently common during the course of development in fungi because they are known to occur in conidia of Botrytis cinerea (Hawker \& Hendy, 1963), ascospores of Neurospora tetrasperma (Lowry \& Sussman, 968) and B. emersonii zoospores (Reichle \& Fuller, 1967). Furthermore, the mitochondria are found during the encystment period dispersed and lying close to the cell membrane, suggesting that they function in cell wall synthesis during the period of mycelial growth.

The presence of the concentric granule, Pl. 4, fig. II and I2, apparent in various stages of the life-cycle (Skucas, 1968) except the gametes (Blondell \& Turian, 1960), is of interest because it seems to be found only in the genus Allomyces among the aquatic Phycomycetes that have been studied with the electron microscope. Its 
Journal of General Microhiology, Vol. 56, No. 2

Plate I

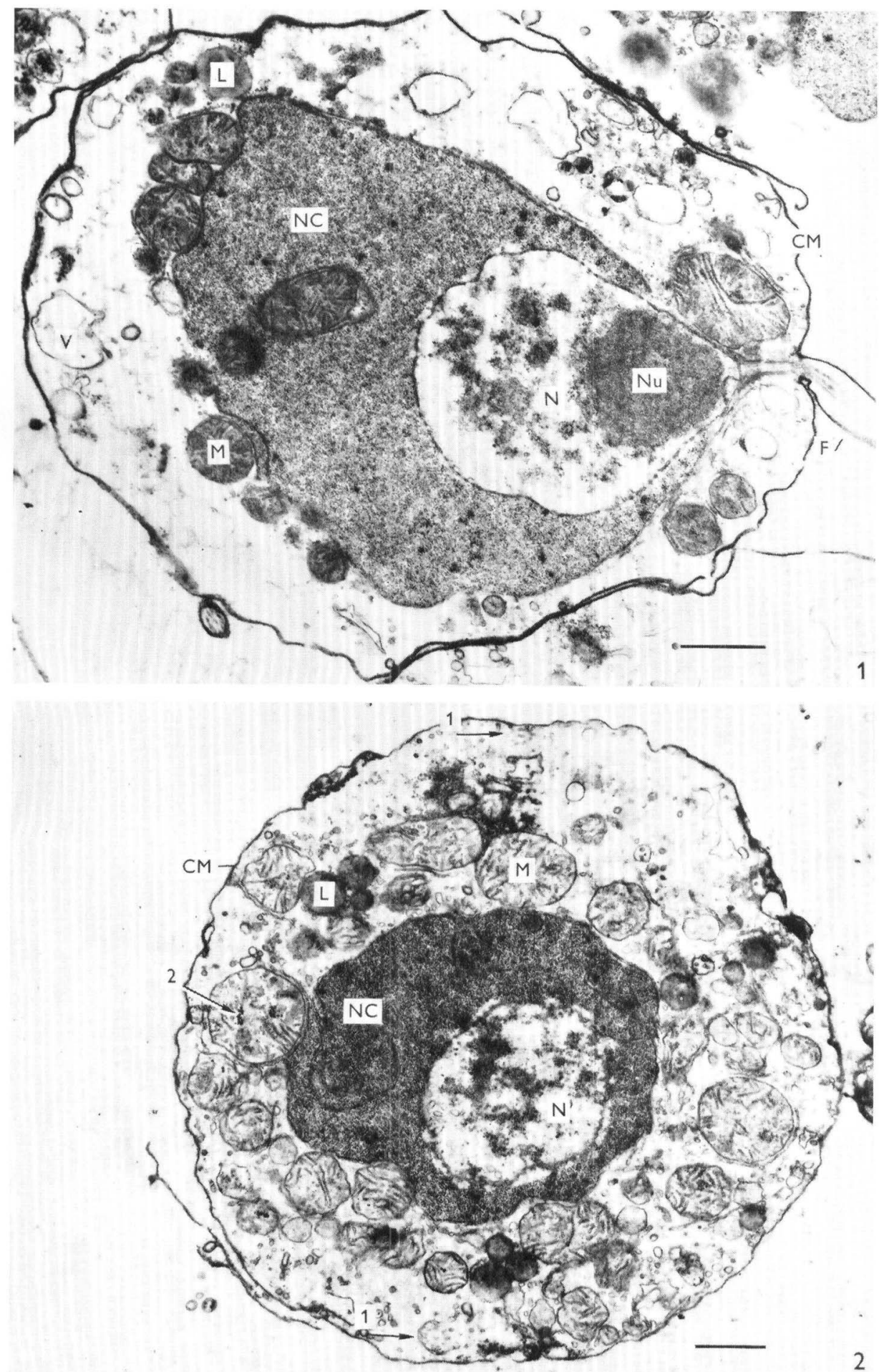

F. P. HIL L 

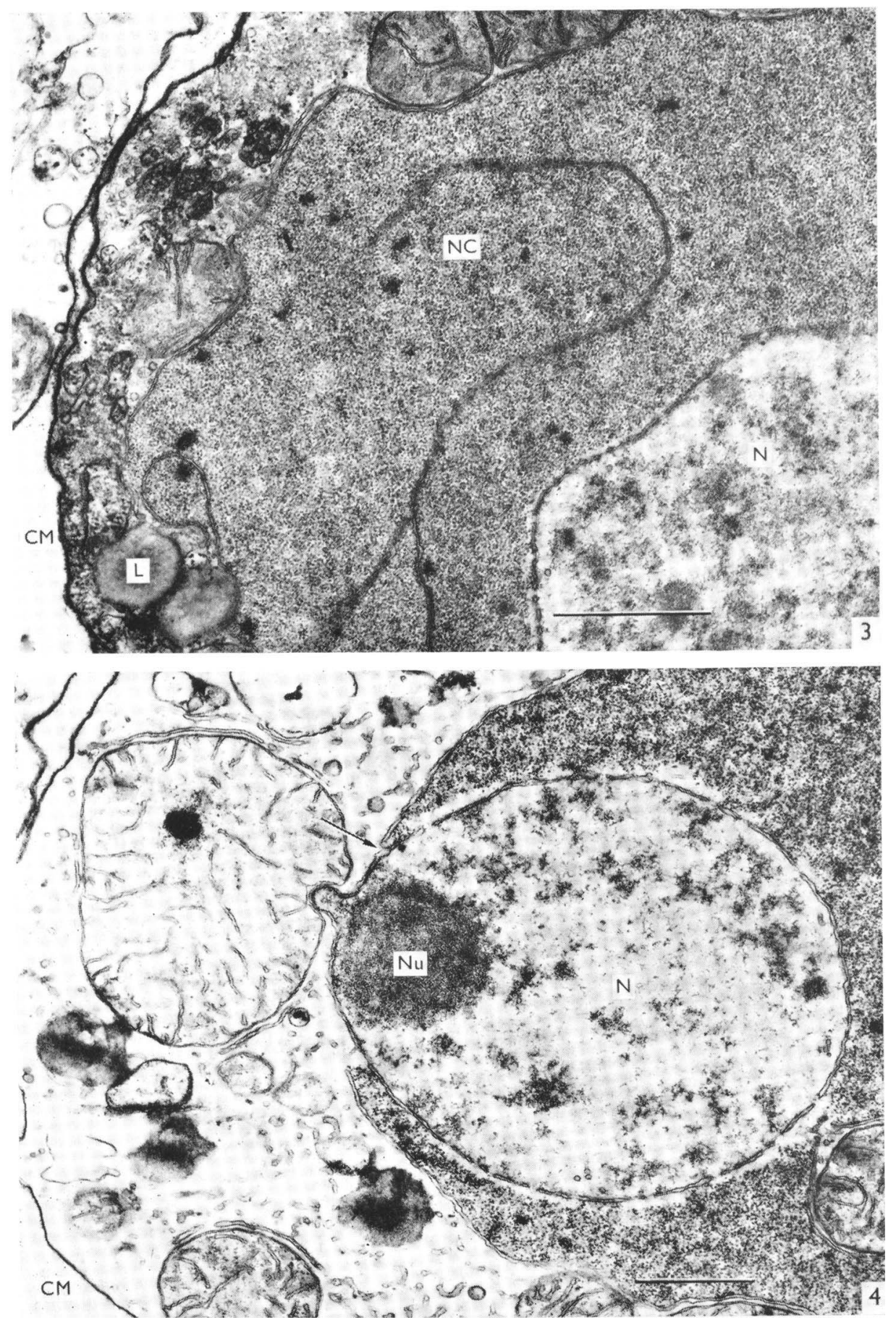

E. P. HILL 

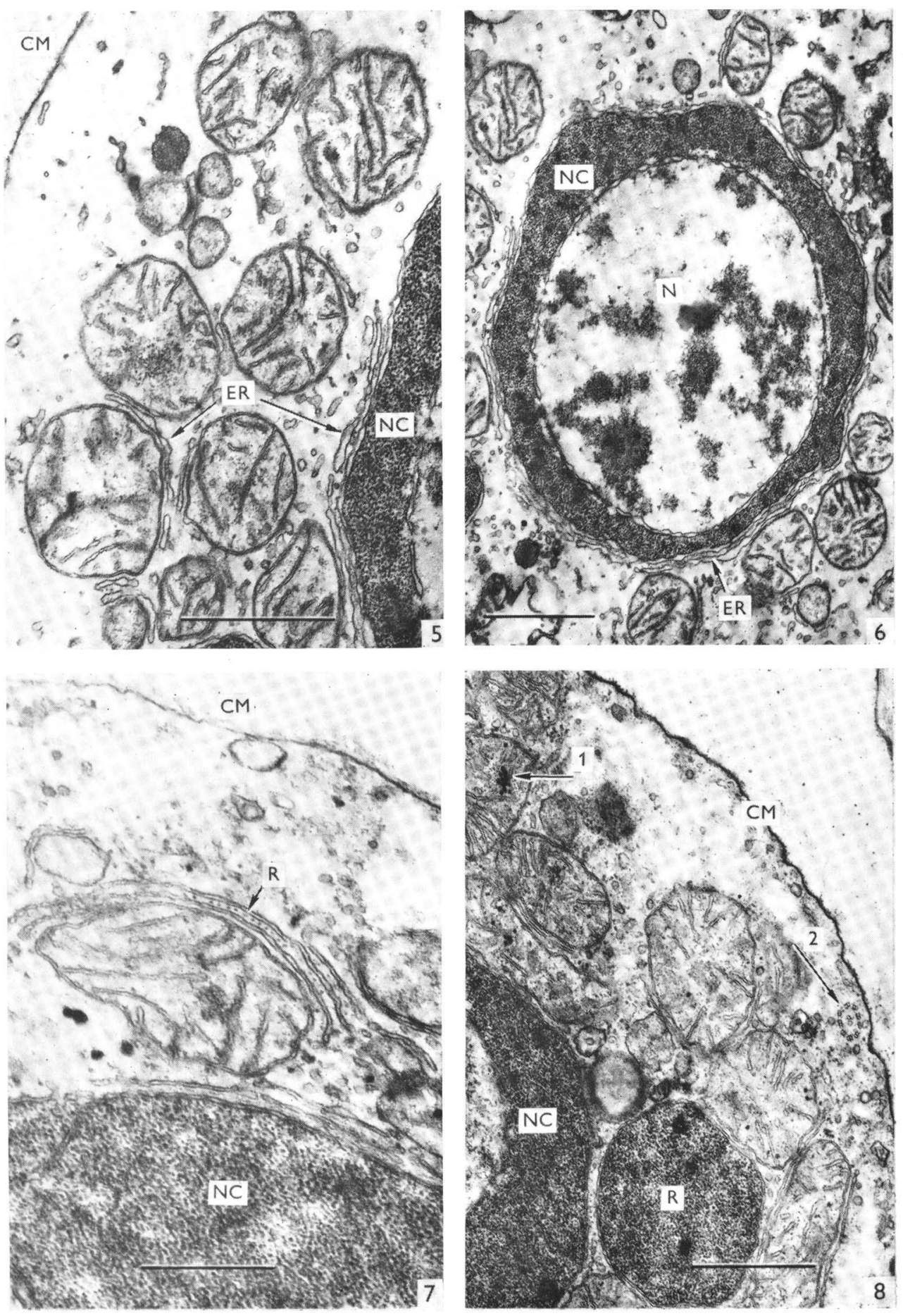

E. P. HILL 

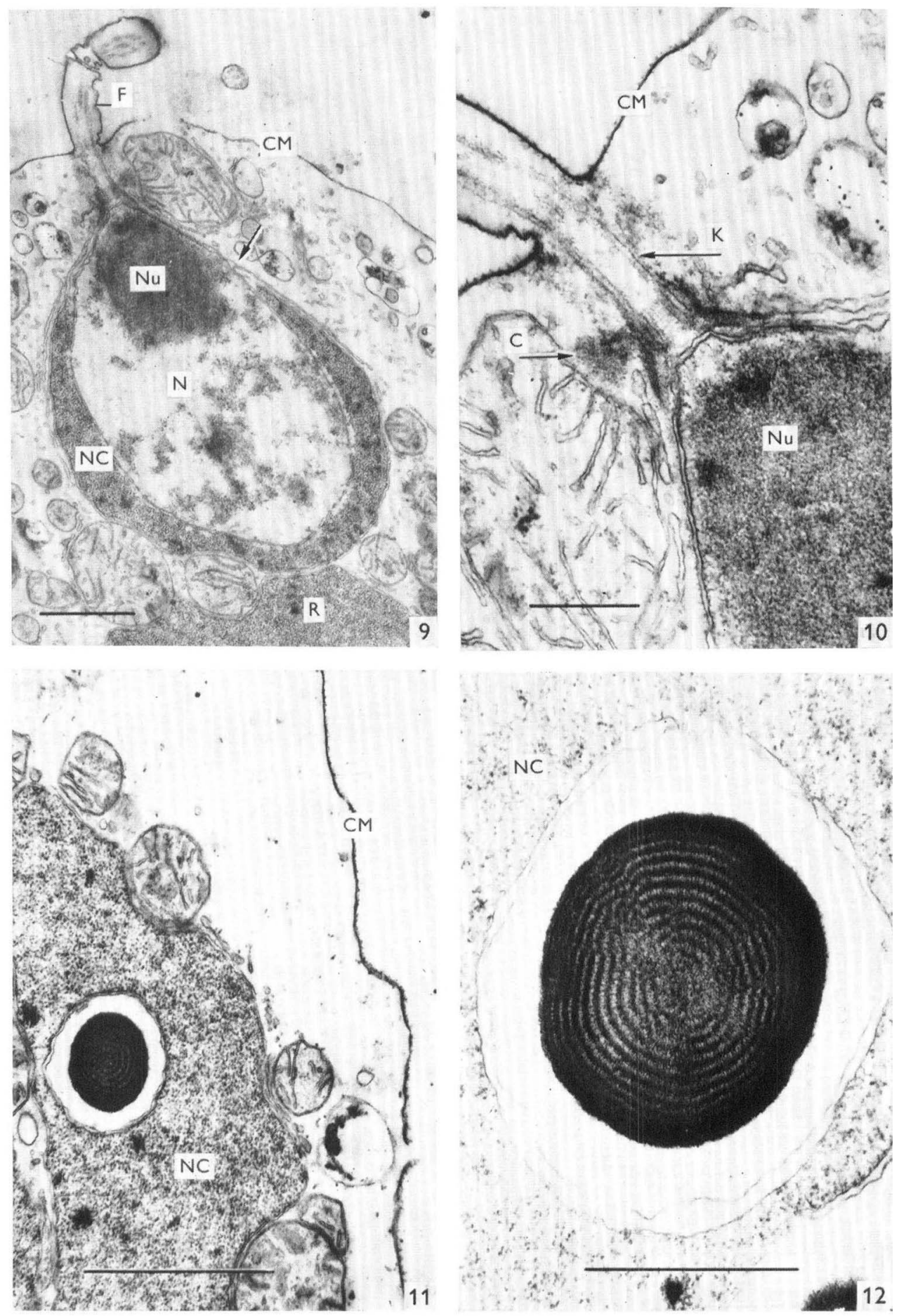

I.P. HIIL 

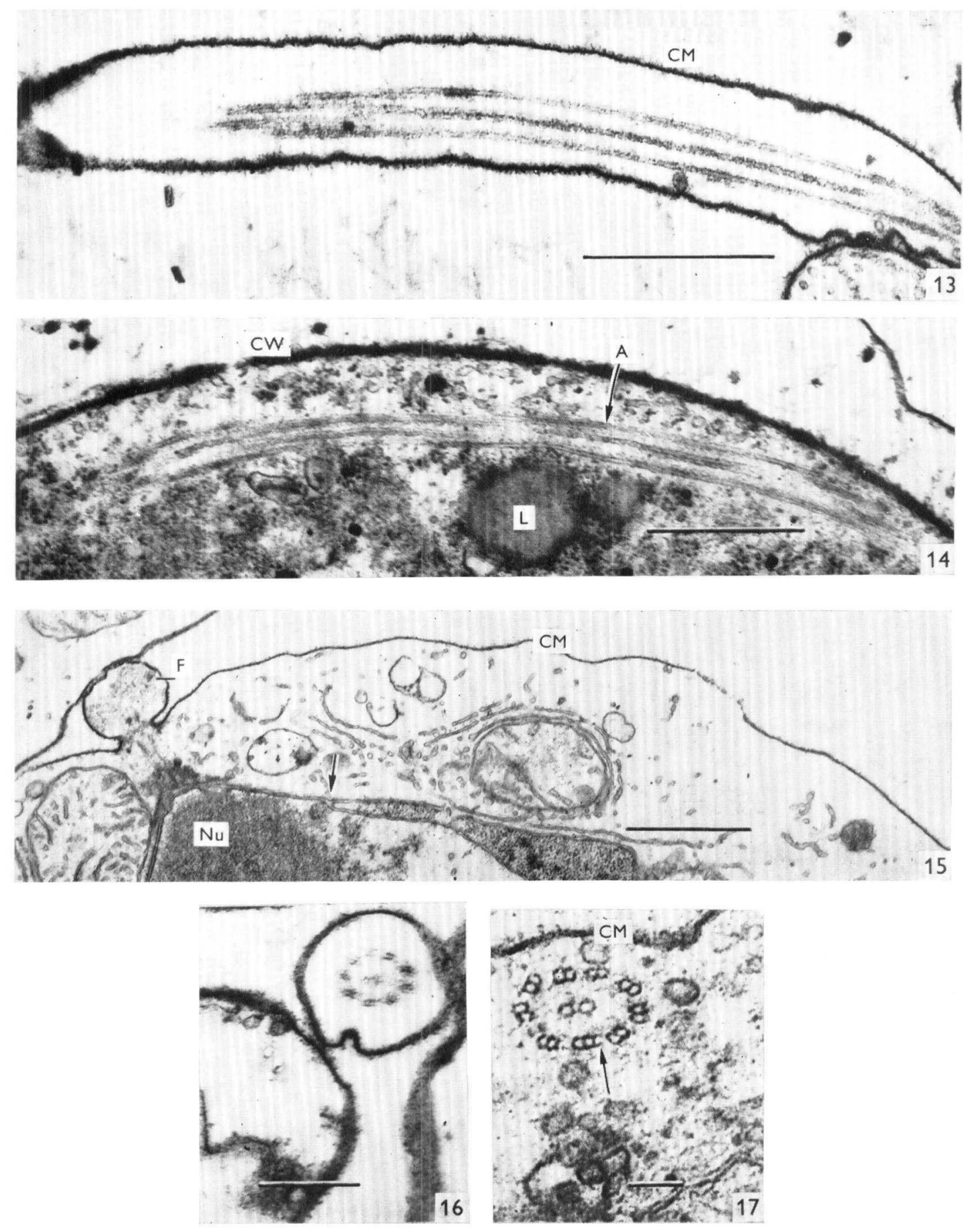

F. P. HIII 
composition, function and formation are not known, although it may be a site of accumulation of metabolic products. Finally, the appearance of granules found only in the mitochondria of encysted spores (Pl. I, fig. 2) are very similar to the polysaccharide granules reported by Lessie \& Lovett (I968) during the formation of Blastocladiella emersonii zoospores.

The formation of the membrane that surrounds the nucleus and nuclear cap, the position and number of centrioles and the nature of the change, if any, that takes place during the time just after the zoospore is released, and its appearance in the sporangium just prior to its release, are points which are currently being studied.

This work was supported, in part, by the Macalester College research fund. The excellent technical assistance of James Barron is gratefully acknowledged.

\section{REFERENCES}

Blondell, B. \& TuRIAN, G. (1960). Relation between basophilia and fine structure of cytoplasm in the fungus Allomyces macrogynus Em. J. biophys. biochem. Cytol. 7, 127.

Chambers, T. C., Markus, K. \& Willoughby, L. G. (1967). The fine structure of the mature zoosporangium of Nowakowskiella profusa. J. gen. Microbiol. 46, I35.

EMERSON, R. ( 1958). Mycological organization. Mycologia 50, 589.

Fuller, M.S. (I 966). Structure of the uniflagellate zoospores of aquatic Phycomycetes. In The Fungus Spore, I 8th Symposium Colston Research Society. London: Butterworth.

FULLER, M. S. \& ReICHLE, R. (I965). The zoospore and early development of Rhizidiomyces apophysatus. Mycologia $\mathbf{5 7}, 946$.

Fuller, M. S. \& Reichle, R. E. (I968). The fine structure of Monoblepharella sp. zoospores. Can. J. Bot. 46, 279.

HAWKER, L. E. \& HENDY, R. J. (I963). An electron-microscope study of germination of conidia of Botrytis cinerea. J. gen. Microbiol. 33, 43.

Kole, A. P. (1965). Flagella in the fungi: An Advanced Treatise, vol. I. New York and London: Academic Press.

Lessie, P. E. \& LovetT, J. S. (I968). Ultrastructural changes during sporangium formation and zoospore differentiation in Blastocladiella emersonii. Am. J. Bot. 55, 220.

LOWRY, R. J. \& SUSSMAN, A. S. (1968). Ultrastructural changes during germination of ascospores of Neurospora tetrasperma. J. gen. Microbiol. 51, 403.

Reichle, R. E. \& Fuller, M. S. (1967). The fine structure of Blastocladiella emersonii zoospores. Am. J. Bot. 54, 8I.

Renaud, F. L. \& Swift, H. (1964). The development of basal bodies and flagella in Allomyces arbusculus. J. Cell Biol. 23, 339.

REYNolds, E. S. (1963). The use of lead citrate at high $\mathrm{pH}$ as an electron-opaque stain in electron microscopy. J. Cell Biol. r7, 208.

SkuCAs, G. P. (1968). Changes in wall and internal structure of Allomyces resistant sporangia during germination. Am. J. Bot. 55, $29 \mathrm{I}$.

\section{EXPLANATION OF PLATES}

Abbreviations used. A, axoneme; C, centriole; CM, cell membrane; CW, cell wall; ER, endoplasmic reticulum; F, flagellum; K, kinetosome; L, lipid body; $M$, mitochondrion; $N$, nucleus; NC, nuclear cap; $\mathrm{Nu}$, nucleolus; $\mathrm{R}$, ribosomes; $\mathrm{V}$, vacuole.

The bar represents I $\mu$ unless otherwise indicated. All magnifications are approximate.

\section{Plate I}

Fig. I. A median longitudinal section through a motile spore.

Fig. 2. A near median section through an encysted spore. Arrow I indicates a cross-section of the retracted axoneme. Arrow 2 indicates polysaccharide bodies in mitochondrion. 


\section{Plate 2}

Fig. 3. Enlarged portion of an encysted spore. Note endoplasmic reticulum dissecting nuclear cap.

Fig. 4. Enlargement of a portion through the posterior area of a motile space. Arrow indicates continuity of nuclear cap and nuclear membrane.

\section{Plate 3}

Fig. 5. A portion of an area of mitochondria from a motile spore. Note spherical shape.

Fig. 6. A cross-section through the nucleus near the anterior end of a motile spore. Arrow indicates abundant endoplasmic reticulum surrounding the nuclear cap.

Fig. 7. A portion of a motile spore showing an enlarged view of ribosomes (arrow) lying between two strands of endoplasmic reticulum. Bar indicates $0.5 \mu$.

Fig. 8. An enlarged section of an encysted spore. Arrow I indicates polysaccharide granules in a mitochondrion. Arrow 2 indicates a cross-section of a retracted flagellum.

\section{Plate 4}

Fig. 9. A section through the posterior third of a motile spore showing the relationship between the flagellum and nucleus. Arrow indicates junction of nuclear and nuclear cap membrane.

Fig. 10. An enlargement of a median longitudinal section showing the region of flagellar attachment. Bar indicates $0.5 \mu$.

Fig. II. A concentric body embedded in the nuclear cap.

Fig. 12. An enlargement of the concentric body.

\section{Plate 5}

Fig. 13. A longitudinal section of the flaygellum.

Fig. 14. A section through an encysted spore showing a longitudinal view of a retracted axoneme (arrow).

Fig. 15. A section through a spore just prior to encystment showing the flagellar bulb. Arrow indicates junction of nuclear and nuclear cap membrane.

Fig. 16. Cross-section of flagellum with the cell membrane surrounding the axoneme. Bar indicates $0.5 \mu$.

Fig. 17. Enlarged view of a retracted flagellum in an encysted spore. Note loss of cell membrane. Arrow indicates arm on one of the doublets. Bar indicates $0 \cdot 1 \mu$. 